\title{
High Precision Optical Surface Topometry
}

\author{
H.J. Tiziani \\ Universităt Stuttgart, Institut für Techn. Optik, Pfaffenwaldring 9, D-70569 Stuttgart, Germany
}

Key Words: Methods and Systems / Optical Metrology and Sensoric / Surfaces / Surface Topometry

\begin{abstract}
For contactless measurement of the surface topography optical techniques are promissing. A lot of progress was made by the development of laser supported methods. It is due to the development of new different lasers, the introduction of solid state detectors and powerful computers for the information processing. Time of flight, phase measurements, active and passive triangulation and projected fringes are very robust techniques for industrial applications.

A lot of progress was made in the application of interferometry to surface and vibration measurements. One or more wavelength together with diode lasers are used. Although interferometry requires polished surfaces, however, by using synthetic wavelengths, it can also be applied for optically rougher surfaces. In addition the unambiguity range will be extended when longer synthetic wavelengths are used.

Furthermore scanning and whole field confocal microscopy can be used for macro- and microstructure analysis. New techniques will be described to extend the field of view.

Some limits especially with respect to resolution and industrial application will be discussed for the methods presented together with some experimental results and future developments.
\end{abstract}

\section{Introduction}

Different noncontact methods are appropriate for microand macrostructure measurements. Introduced are timeof-flight and phase-measuring-techniques with a resolution on the order of a few mm to one mm respectively. Furthermore active and passive triangulation techniques are very robust and appropriate for different applications in precision measurements.

Different techniques can be used for optical 3-D-measurements. They are summarized in Table 1. For the measurement of the topography the principle of triangulation as well as its extension to light sectioning and projected fringe methods can be applied successfully [1, 2].

Moiré techniques or projected fringe techniques are becoming useful tools for topography measurements. They can be applied to determine surface shape or deviation of the shape or vibration amplitudes. The generation of the grating can occur by projecting a ruling or grating upon a surface which is seen through a detecting system with a reference grating. Alternatively a grating pattern can be generated by interference of two plane waves upon the surface.

Furthermore image plane locating systems can be used to be applied to analyse the surface topometry. In the last years confocal principles were studied to be applied for the measurement of the surface geometry.

Today, laser interferometry is probably one of the most commonly used techniques for high resolution measurements in metrology. A stabilized $\mathrm{He}-\mathrm{Ne}$-laser with an absolute stability of better than $10^{-7}$ is frequently used as light source. The accuracy for length measurements is limited by atmospheric conditions (humidity, temperature, pressure) rather than by the laser stability. Furthermore, single mode diode lasers are used in metrology eventhough frequency stability is still a problem especially in interferometry.

Interferometry and Moiré techniques as well as image plane locating systems will be applied more frequently, when used with image processing. They are becoming useful tools for precision measurements in research and for industrial applications. Computer analysis is increasingly important for fringe analysis. The use of solid-state detector arrays, image memory boards together with microprocessors and computers for the extraction of the information from the interferograms and high-resolution graphic boards find important application in optical metrology. Much more information can be extracted from the sensor data, leading to higher sensitivities and accuracies [3].

Table 1

Resolution of optical 3-D-measuring principles

\section{Optical methods}

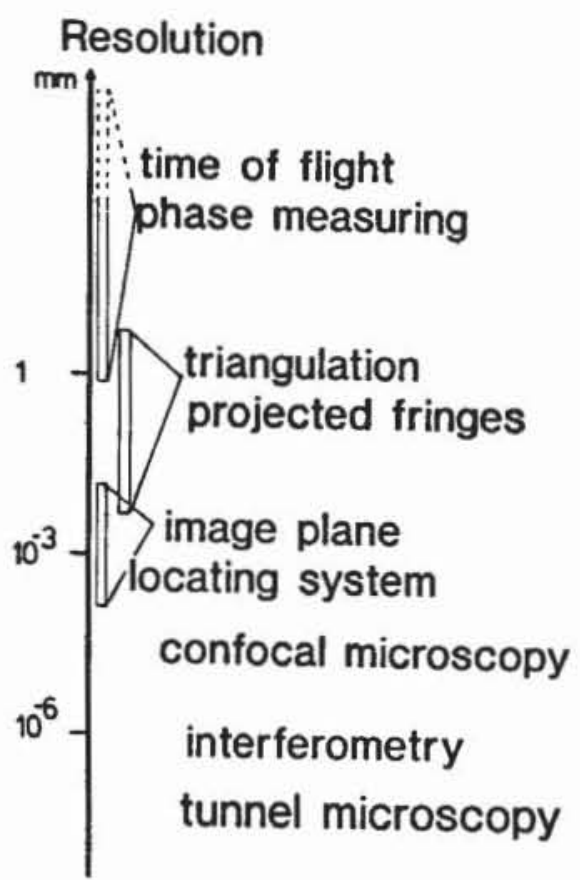


Fringe analysis procedures including fringe peak detection and fringe order determination are tedious and timeconsuming. Automatic fringe analysis and precision phasemeasuring-techniques are very important in applying interferometric techniques. Automatic quantitative evaluation of interferograms requires accurate interference phase measurements, independent of fringe position and intensity variations superposed onto the interferograms. In many interferometric arrangements, phase shifting or heterodyne techniques have been introduced for automatic fringe analysis.

The phase shifting technique is very appropriate for digital processing and TV techniques. Two-beam-interferometry together with video electronic processing lead to a sensitivity of $1 / 100$ of a fringe at any point in the fringe pattern in the TV image. In heterodyne methods the relative phase increases linearly in time and the reference phase is measured electronically at the beat frequency of the reconstructed wavefields. Heterodyne interferometry offers high spatial resolution and interpolation up to $1 / 1000$ of a fringe. It requires, however, sophisticated electronic equipment and mechanical scanning of the fringe pattern.

\section{Triangulation}

Active and passive triangulation techniques are powerful tools for contactless measurements. Passive triangulation is used in photogrammetry to obtain the topography. For active triangulation a laser spot is projected on to the object. Its image position is recorded on a position sensitive detector or on a CCD-chip (line or array camera). The lateral displacement of the spot image is directly related to the depth in the object (Fig. 1). The resolution of the triangulation techniques is given by

$\mathrm{d} z=\frac{\Delta W \cdot z_{0}^{2}}{B}$

where $z_{0}$ is the working distance, $B$ is the base, $\Delta W$ is the angular resolution of the detecting system.

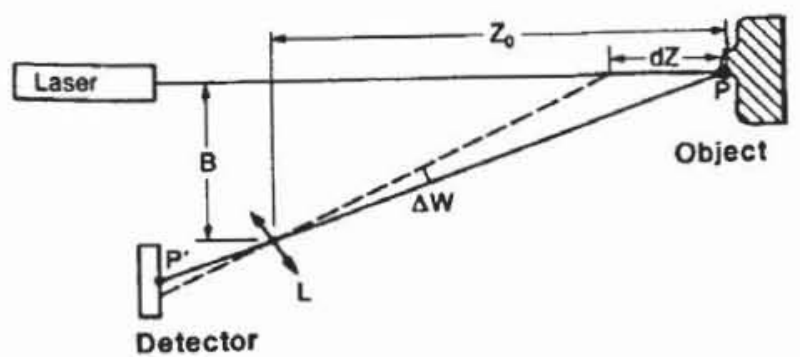

Fig. 1

Principle of static triangulation

Triangulation-based 3-D-sensors are appropriate tools for inspection and measurement in industrial environments. Especially synchronized single-spot-scanners fulfil the high requirements upon range, resolution and robustness. In Fig. 2 the principle of a scanning triangulation system is

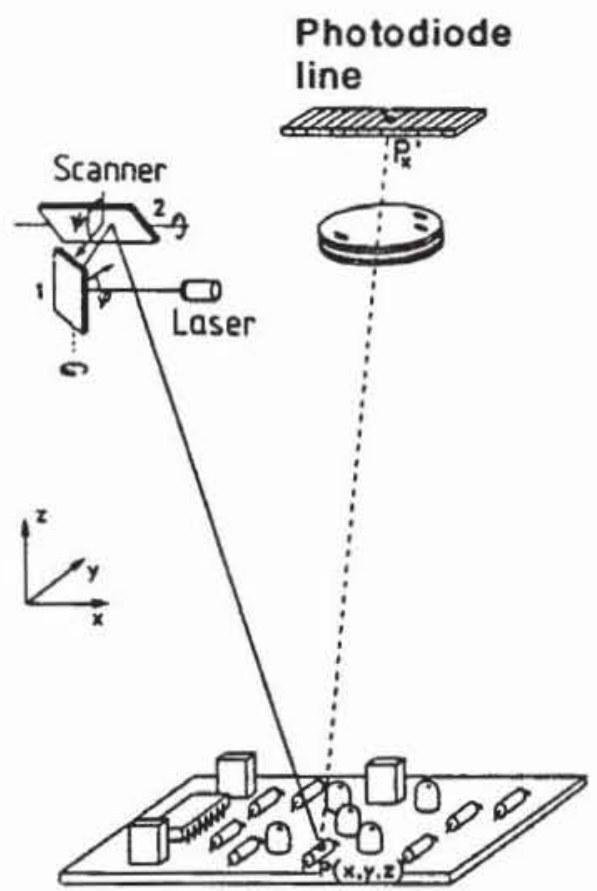

Fig. 2

Principle of dynamic triangulation

shown schematically, the galvano scanner mirrors are controlled by computer to select the $x-y$ coordinates. Angular resolutions of $5 \mu \mathrm{rad}$ can be obtained. The $z$ coordinate is given by the triangulation principle discussed before. Furthermore, a synchronisation of the observation beam with the illumination beam of the scanner may be realized optomechanically, typically by leading the observation beam

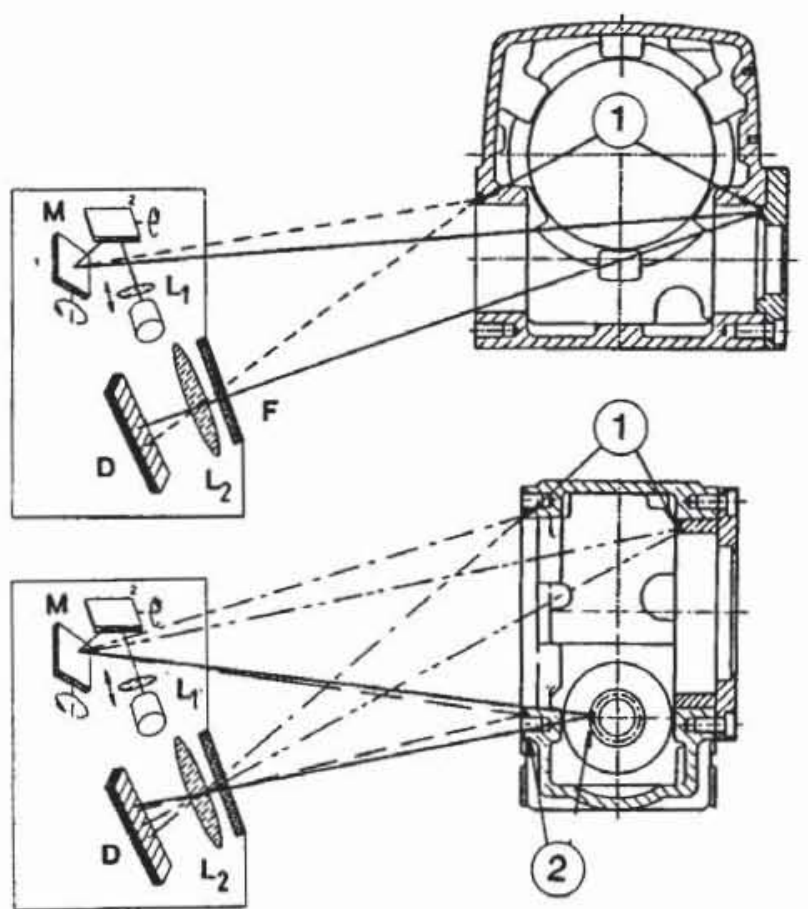

Fig. 3

Measuring of gear box with the dynamic triangulation; $M=$ galvanometer mirrors, $L_{1}=$ focussing lens system, $L_{2}=$ image forming lens system, $\mathrm{F}=$ optical filter, $\mathrm{D}=$ Photodiode line 
by a mirror, which is rigidly connected to the scanning mirror of the illumination beam.

In Fig. 3 the principle of the 3-D-measurement of a workpiece is shown. The measuring time of one point is in the order of $1 \mathrm{~ms}$.

\section{Projected Fringe Techniques for Industrial Inspection}

Light sectioning and projected fringes are an extension of the triangulation for out of plane and in plane displacement - and for deformation measurements and topography. Projected fringe patterns can be formed by different methods such as projecting either a grating like structure or an interference pattern. Height variations or deformations lead to a deformation of the projected fringes, which in turn are compared with the original or synthetic grating-like structure. Typical contour-line separations can vary between micrometer and millimeter.

Moiré techniques can therefore also be used for microsurface analysis as will be shown.

In Moiré techniques a deformed grating structure is superimposed onto the original grating. The grating structures do not need to be resolved. The topography, deformation and vibrations can be analysed.

Fig. 4 a shows an arrangement used at our Institute where the fringes are produced by interference due to a relative tilt

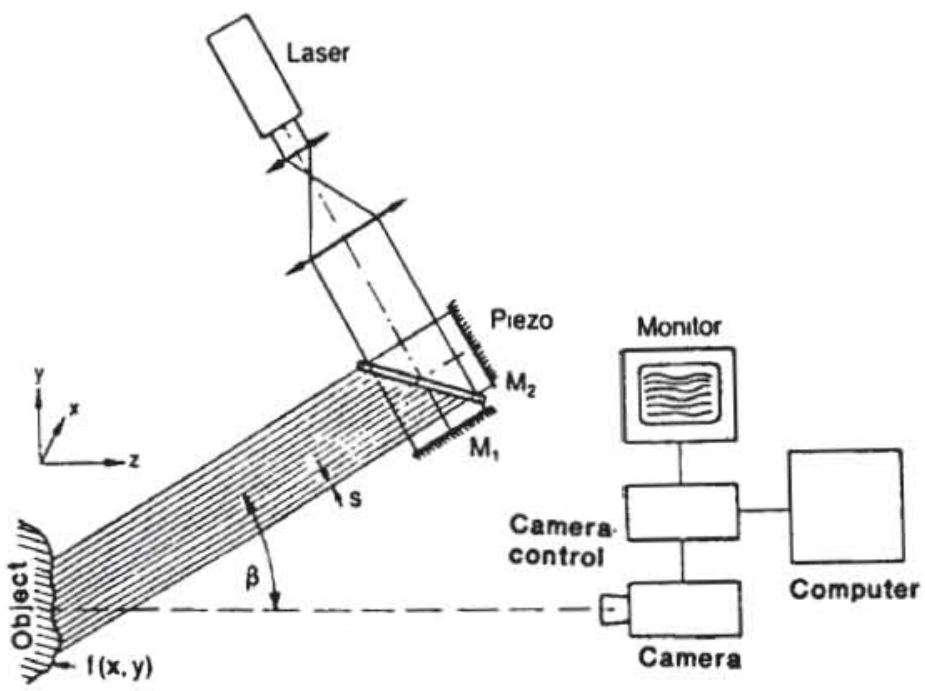

(a)

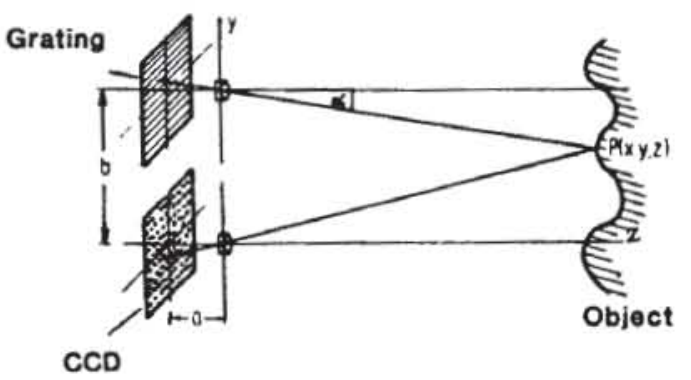

(b)

Fig. 4

Principle of the projected fringes, a) fringe generation by interference, b) grating projection between the mirrors $\mathrm{M}_{1}$ and $\mathrm{M}_{2}$. The line separation can be adapted depending on the application by tilting the mirror $\mathbf{M}_{1}$. For the analysis of steps at least two grating periods are needed, a coarse one for the absolute height measurement and by contrast a fine one for the precision measurement. A piezo-element is used to shift the phase for the analysis of the deformed fringes using one of the phasestepping techniques to be described. By changing the phase, the fringes on the object will move in steps of, say, onequarter of the period. In, Fig. 5 it is shown that from the fringe deformation due to the shape of the cornea of the eye a pseudo $3 \mathrm{D}$ plot can be obtained. Alternatively the gratinglike structure can be projected onto the object by using a grating or generating a grating with liquid crystal cells. Fig. 6 shows the results of an arrangement where grating structures were generated in liquid crystals and projected onto the object.

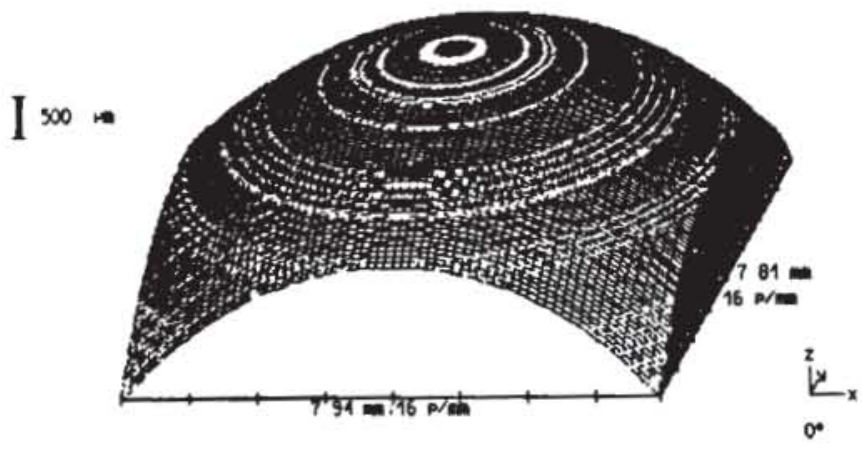

Fig. 5

Results of projected fringes onto the simulated cornea of the eye in pseudo 3-D representation

So far 640 grating-like structures could be generated in Fig. 6, where 320 lines were used. The fringe analysis to obtain the topography occurs by phase shifting, i.e. by lateral shifting of the grating structure, for instance, in steps of a quarter period each.

A number of techniques have been developed for microand macrotopography. The choice of an appropriate technique depends on the sensitivity required, which in turn can be adjusted. Moiré techniques and projected fringe techniques are not as sensitive as interferometric and holographic techniques. The sensitivity of the structured light technique can, to some extend, be adapted to the requirements by selecting the appropriate fringe spacing. A similar adaption is hardly possible in interferometry or holography and furthermore the projected fringe and Moiré techniques are less sensitive to disturbances, such as vibrations than interferometry and holography.

Real-time Moiré techniques are also useful for detecting vibration patterns as well as for identifying different kinds of motions. To improve the robustness and resolution different structures such as binary, sinusoidal, triangular and trapezoidal were analysed. Trapezoidal structures were found to be the most appropriate for our gray code projecting system. Furthermore, it was found important to adapt 
the brightness of the projected structure to the local brightness of the object.

To fulfil the different requirements, a pattern generator was constructed by means of a light-emitting diode and a computer-driven galvano scanner. Up to 3000 lines can be generated and modified in quasi real time to be adapted in phase, frequency and brightness. In Fig. 7 different patterns for the same object region are shown, that are delayed in time.

\section{Micro Shape and Roughness Measurement}

3D-shape analysis by fringe projection can become an important method for microstructure analysis. A fringe
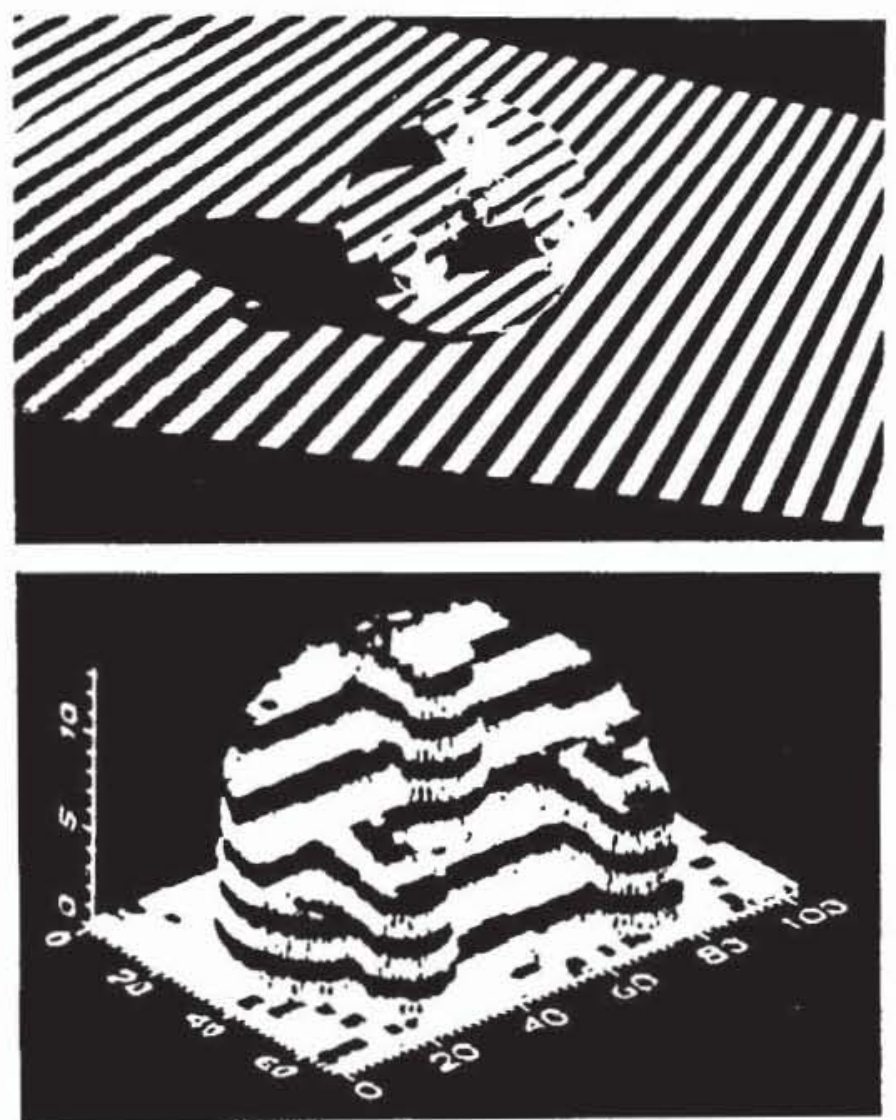

Fig. 6

Results of grating structure generated with a liquid crystal device

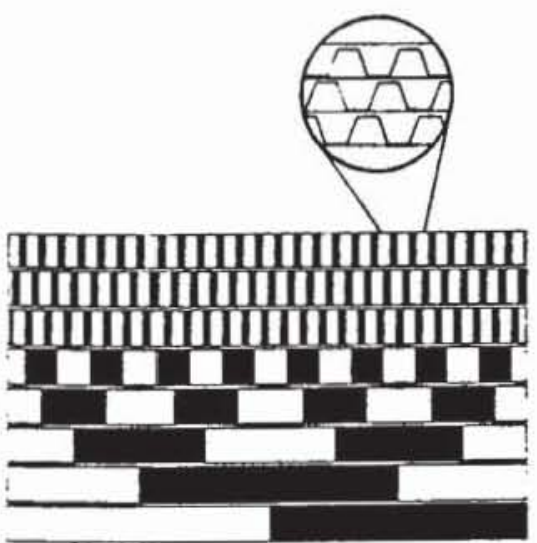

Fig. 7

Time-space diagram of grating structures with hybrid code, generated by the pattern generator projecting microscope, FPM is shown in Fig. 8, where the projection of the grating as well as its image formation on the CCD camera is performed with the same objective, MO. High lateral and vertical resolution of the order of $1 \mu \mathrm{m}$ and $0.1 \mu \mathrm{m}$ respectively can be obtained.

In Fig. 8 the spatially filtered spectrum of a binary line grating (Ronchi grating) is projected into the entrance pupil EP of the microscope objective MO. The grating needs to be projected obliquely onto the object. The spectrum is therefore shifted off-axis (laterally) by the distance $d$, so that the principal rays corresponding to the zero-order of the spectrum of the projected grating form an angle $\beta$ with the optical axis of the microscope objective. As the entrance pupil of a high aperture microscope objective is not physically accessible for spatial filtering, the filtering is done in plane F of Fig. 8, which is conjugated to the entrance pupil EP via the beam splitter and lens O1. This secondary pupil is easily accessible for filtering manipulations.

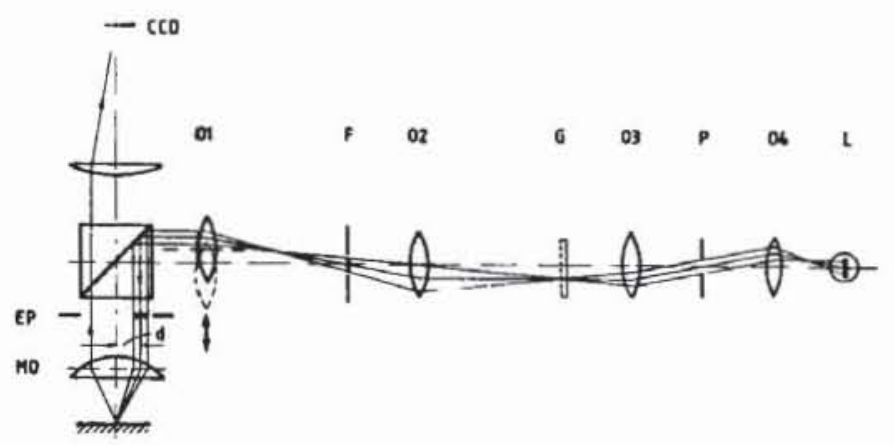

Fig. 8

Fringe projecting microscope

The projecting grating microscope extends the range of applications. It will especially be applied where interference techniques cannot be applied such as for rough surfaces (mean roughness $>0.2 \mu \mathrm{m}$ ). Typical objects are very rough surfaces like sheet-metal, ground and turned metal surfaces, plastics, ceramics, even biological surfaces can be topographed. In addition the topography method can also be used for smooth surfaces like glass and semiconductor surfaces, lacquer and coated surfaces as well as polished and diamond turned surfaces.

A symmetrical configuration with $\pm \beta$ for the directions of projection and imaging according to Fig. 8, leads to an improvement of the depth resolution. The amount of the off-axis shift $d$ is matched to the pupil diameter $D$ in such a way, that the outer first order of the grating spectrum can pass the entrance pupil and sufficient clearance is given for lateral displacement or dispersion of the spectrum of the back-travelling light reflected from inclined or rough surface elements.

In our telecentric FPM-arrangement both the entrance pupil of the imaging system and the exit pupil of the grating projection system lie at infinity in order to compensate for variations in height sensitivity. If the grating is adjusted perpendicularly to the optical axis in plane $G$, the image 
plane of the projected grating is oriented parallel to the $x, y$-plane of the object, and the fringe contrast is constant over the object field.

The grating can be illuminated by a tungsten halogenide lamp $\mathrm{L}$ via lenses $\mathrm{O} 4$ and $\mathrm{O} 3$. The grating structured image distorted by the object topography is formed onto the CCD interline transfer camera with $756 \times 581$ picture elements by the objective Oc. The conversion of the height variation $\Delta h$ due to a local fringe displacement $\Delta x$ in $x$-direction is, for grating projecting angle $\beta$ and an observing angle $-\beta$ :

$\Delta h=\frac{\Delta x}{2 \sin \beta}$.

A typical measurement of an optically rough surface, a metal sheet, is shown in Fig. 9, the mean roughness is $R_{\mathrm{a}}=1.59 \mu \mathrm{m}$.

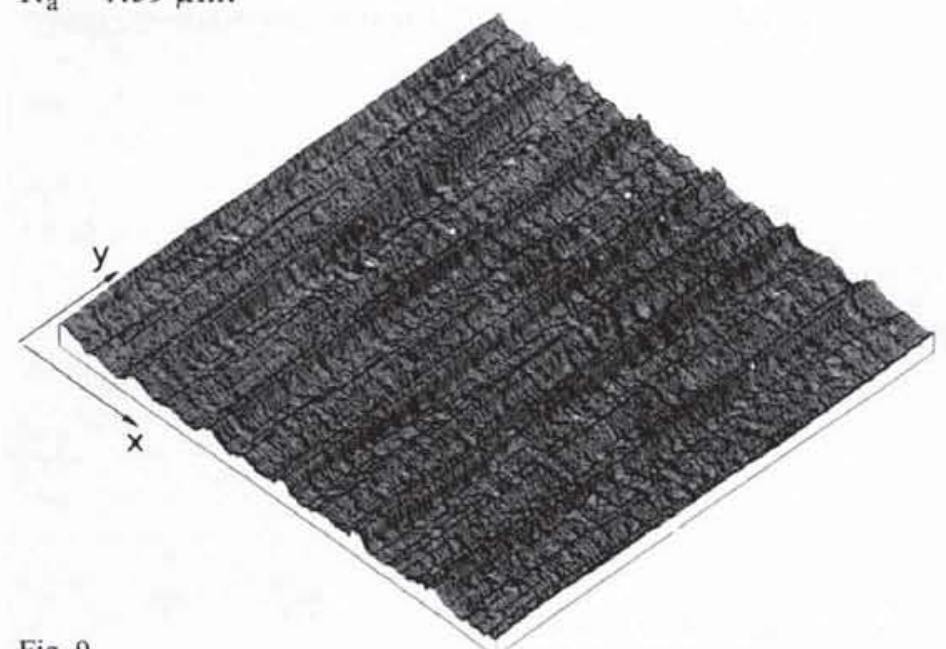

Fig. 9

Surface topography of a metal sheet surface with the mean roughness $R_{\mathrm{a}}=1.59 \mu \mathrm{m}$, obtained with the fringe projecting microscope

\section{Interferometry for Precision Measurements}

The introduction of the laser in 1960 and the progress made recently in automatic fringe analysis are mostly responsible for the widespread application of interferometry in industry today. Two-beam interferometry with a few applications will be discussed.

Fringe analysis is not only important in interferometry, but also in holography, speckle applications and Moiré techniques. The coherent superposition of the two wavefields $a_{1} \cos \left[\omega t-\phi_{1}(x)\right]$ and $a_{2} \cos \left[\omega t-\phi_{2}(x)\right]$ leads to the intensity

$$
I(x)=I_{0}[1+m \cos \phi(x)]
$$

where $\phi(x)=\phi_{1}(x)-\phi_{2}(x)$ is the phase difference, $I_{0}=\sqrt{\left|a_{1}\right|^{2}+\left|a_{2}\right|^{2}}$ the mean intensity and

$m=\frac{2\left|a_{1}\right|\left|a_{2}\right|}{\left|a_{1}\right|^{2}+\left|a_{2}\right|^{2}}$

$m$ being the visibility of the interference fringes.
The phase $\phi$ can be determined from the detector signal of an interference arrangement. In Eq. (2) there are three unknowns $\left(I_{0}, m\right.$ and $\left.\phi\right): \phi$ is determined to a factor of $2 \pi$ only; and sign $\phi$ is to be determined. In addition, the accuracy can be drastically increased by an appropriate interpolation technique. Digital interferometry provides the means for obtaining very precise measurements at rapid rates.

Automatic quantitative evaluation of interferograms requires accurate interference phase measurements, independent of fringe position and intensity variations superposed onto the interferograms. For the fringe analysis, static and dynamic techniques are used whereas in dynamic techniques, an active phase shifting in the interference arrangement is required; this is not necessary in static methods. In many interferometric arrangements, however, phase shifting or heterodyne techniques have been introduced for automatic fringe analysis.

In the phase shifting technique or quasi-heterodyne technique the relative phase is changed continuously or stepwise, using at least three phase shifts of $90^{\circ}$ or $120^{\circ}$, for instance. The phase of the interference patterns can then be computed from the different measured intensity values. Very frequently 5 interferograms are analysed with 4 phase shifts between. The phase-shifting technique is very appropriate for digital processing and TV techniques. Twobeam-interferometry together with video electronic processing lead to a sensitivity of $1 / 100$ of a fringe at any point of the fringe pattern in the TV image. In heterodyne methods, the relative phase increases linearly in time and the reference phase is measured electronically at the beat frequency of the reconstructed wavefields. Heterodyne interferometry offers high spatial resolution and interpolation up to $1 / 1000$ of a fringe. It requires, however, sophisticated electronic equipment and mechanical scanning of the fringe pattern.

\subsection{Fringe Analysis with Static Techniques}

For fringe analysis using static methods, a tilt is often introduced to avoid closed fringes. The fringe centres can be found manually by using a digitizing tablet as well as by using video- and image-processing techniques.

To estimate fringe peaks, and fringe density, binarization technique is commonly used in many fringe analysis systems because of the simple algorithms used. The grey-level method, where the local variation of fringe density is considered, is sensitive to noise; but it can detect peaks by processing only local areas smaller than those in the binary case. In order to extract fringe peaks in the grey-level method, it is very important to diminish the influence of noise, including speckle noise. A simple and effective way is unweighted averaging.

Carrier fringe analysis in the spatial domain using Fourier techniques can be very useful. The Fourier-transformation technique was first proposed by Takeda et al. [5] and extended afterwards by different worker $[3,5]$. 


\subsection{Fringe Analysis with Dynamic Techniques}

For dynamic algorithms the relative phase between the reference beam and the test beam in an interferometer is varied at a constant, controlled rate or in steps of $90^{\circ}$ or $120^{\circ}$ for instance. Dynamic techniques are: phase shifting in three, four or more steps, or continuously, the heterodyne technique and the phase-locked technique $[3,6]$.

\subsubsection{Phase-shifting Techniques}

The intensity of the interference pattern can be written as

$I(x, y)=I_{0}(x, y)\left[1+m(x, y) \cos \left[\phi(x)+\Delta_{k}\right]\right]$

where $\phi(x, y)=\frac{2 \pi}{\lambda} W(x, y)$ is the phase distribution of the wavefront $W(x, y)$ across the interference pattern to be measured and $\Delta_{k}$ is the deliberately introduced phase shift. $k=1,2,3 \ldots N$, depending on the number of phase shifts introduced.

The interference pattern can be recorded by a solid-state detector array. For the shifting technique, at least three patterns with the appropriate phase shifts need to be recorded. Applying the four-phase shift method $(-3 \pi / 4,-\pi / 4$, $+\pi / 4,+3 \pi / 4)$, the phase of the wavefront computed from the four interferograms is

$\phi(x, y)=\arctan \frac{I_{4}(x, y)-I_{2}(x, y)}{I_{1}(x, y)-I_{3}(x, y)}$.

For accurate phase analysis, the phase shifts introduced by a piezo-element, for instance, need to be measured for the piezo to be calibrated. An error of $\lambda / 10$ in the wavefront occurs by an error in the phase shift of $20 \%$. Care needs to be taken when phase shifts in strongly diverging or converging beams are necessary. Schwider et al. (7) proposed a four-step phase shift procedure with an averaging algorithm, which leads to a reduction of errors introduced by inaccurate phase shifts. Furthermore the phase stepping could be anything between $0^{\circ}$ and $180^{\circ}$. By the integrating bucket technique the intensity of the fringe pattern is integrated in buckets while the phase is being shifted $[3,6,7]$.

For high-accuracy measurements of flat and spherical surfaces, it is useful to work with few fringes: a phase calculation algorithm with five measurements of intensity is desirable. The corresponding phase steps are $-2 \Delta,-\Delta, 0$, $\Delta$ and $2 \Delta$. For $\Delta=90^{\circ}$

$\tan \phi=\frac{2\left(I_{2}-I_{4}\right)}{2 I_{3}-I_{5}-I_{1}}$.

The preliminary calibration of the piezo to ensure that the phase step is approximately equal to $90^{\circ}$ can be carried out with four out of the five values of intensity available, namely

$\cos \Delta=\frac{I_{5}-I_{2}}{2\left(I_{4}-I_{2}\right)}$.
Because the five-interferogram method is not strongly affected by phase stepping errors, it is particularly interesting in a Fizeau arrangement where, for different aperture heights, different phase shifts occur while shifting the test surface or the reference.

Digital interferometry is very useful for getting the interferometric data into a computer for the analysis. An experimental arrangement with oblique incidence is shown in Fig. 10. For the automatic analysis of closed fringes a phase-shift technique is appropriate. Phase shifts can be introduced by tilting a plane-parallel plate, moving a mirror by means of piezo-elements or by polarization techniques.

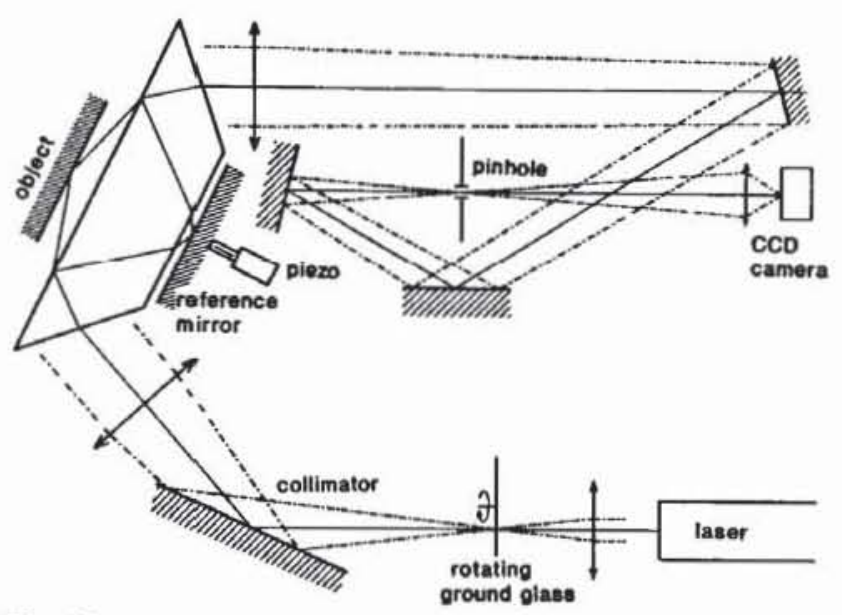

Fig. 10

Arrangement for interferometry with oblique incidence

\section{Interferometry at Oblique Incidence}

Interferometry is a powerful tool for high resolution measurement of the topography of polished surfaces. It can, however, not be applied to study and measure the topography of optical rough surfaces. Height variations $\Delta z$ of the object will change the phase $\phi$ of the reflected object beam. This phase variation detected by an interferometer, for instance, can be measured as a function of position $x$ on the surface. A problem occurs when the surface has step height variations greater than $\lambda / 2$, in reflection. A discontinuous height variation $\Delta z$ introduces a phase jump $\Delta \phi$ given by $\Delta \phi=(4 \pi / \lambda) \Delta z$. However, the interferometer can only determine the phase $\phi$ modulo $2 \pi$. A reduction in sensitivity is obtained by an oblique incidence of the wavefront onto the test object. The optical path difference in air is $W=2 n \Delta z \cos \theta$, where $\Delta z$ is the distance between the reference surface and the object in a Fizeau arrangement and $\theta$ the angle of incidence onto the object. The optical sensitivity is therefore reduced by $\cos \theta$. For $\theta=81^{\circ}$ the effective or synthetic wavelength is $4 \mu \mathrm{m}$ for a $\mathrm{HeNe}$ laser source with $\lambda=633 \mathrm{~nm}$. In Fig. 10 the incident beam is separated by the prism surface into the reference and object beam with oblique incidence. The reference wave is phase shifted by the piezo driven reference mirror before the reference and object beam are combined. The fringe pattern is projected onto the CCD chip. The special arrangement avoids multiple reflections and image distortions. The result 


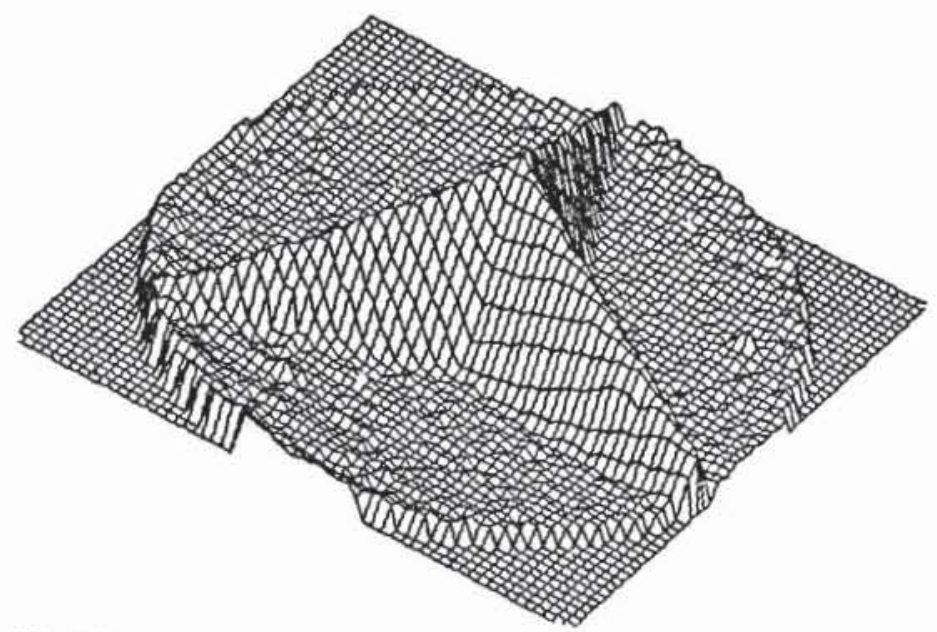

Fig. 11

Topography in pseudo 3D-representation of a copper workpiece obtained with $\lambda_{\text {eff }}=4 \mu \mathrm{m}, \mathrm{PV}=3.4, \mathrm{RMS}=0.58 \mu \mathrm{m}$

of the analysis of a copper surface is shown in Fig. 11. The depth resolution is in the order of $0.08 \mu \mathrm{m}$, the fringe analysis occurs in a few seconds.

\section{Interference Microscopy}

For microprofile measurements, stylus instruments are among the most highly developed means of profiling precision surfaces. Interferometry with fringe analysis can be used for microstructure analysis. An adapted TwymanGreen interference arrangement with high numerical aperture is shown in Fig. 12 (Linnik Type). The object and

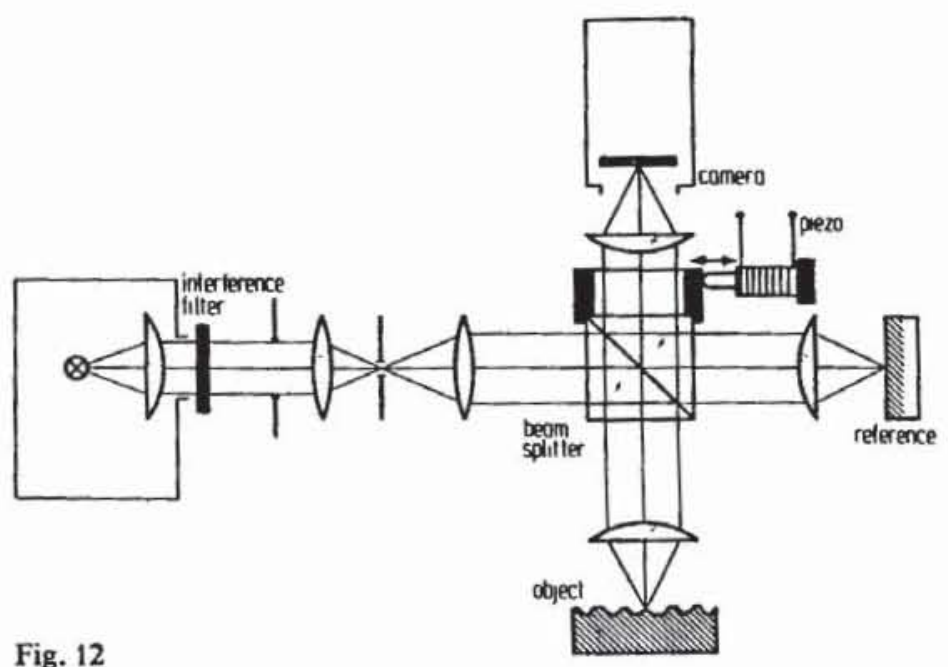

Arrangement for interference microscope

reference beam are superimposed after the beam splitter and the image of the surface under test, where the fringes are localized near the test object are imaged on to a CCD camera. A high numerical aperture objective is used to obtain high lateral and subnanometer depth resolution. A piezo-element shifts the phase in the reference beam (objective and mirror) in steps. A diode array of $500 \times 580$ elements was used to study the compact disc with a pitch of $0.6 \mu \mathrm{m}$ as shown in Fig. 13. The numerical aperture, NA, of the objective was NA $=0.9$.

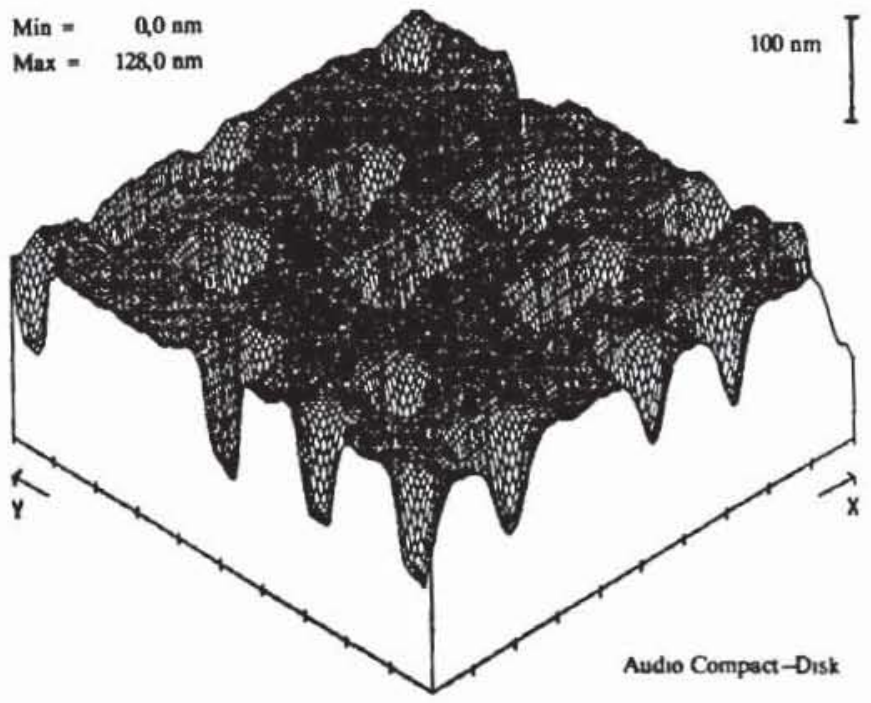

Fig. 13

Interferometric analysis of a compact disc with a pitch size of $0.6 \mu \mathrm{m}$ and a depth of $120 \mathrm{~nm}$

Often it is useful to describe the wavefront by polynomials. For circular aperture, Zernike polynomials are preferred because of their orthogonal properties. For special applications, Zernike-Tatian polynomials (for a system with a central obstruction) or Tschebyscheff polynomials are useful.

\section{Heterodyne Interferometry}

In interferometry, phase differences of optical fields are transformed into detectable intensity variations. In heterodyne interferometry the time-dependent phase variation is analysed in the frequency space. The two light fields are assumed to be

$A_{1}=a_{1} \cos \left[\omega t+\phi_{1}(x, y)\right]$

$A_{2}=a_{2} \cos \left[\omega t+\Delta \omega t+\phi_{2}(x, y) \pm \phi(x, y, t)\right]$

where $\Delta w$ is proportional to the frequency shift $f=f_{2}-f_{1}$ and $\phi(x, y, t)$ is the time-varying phase shift leading to $\mathrm{a}$ frequency shift by interference of the two wavefields:

$$
\begin{aligned}
I(x, y)= & I_{0}\{1+m(x, y) \cos [\Delta \omega t \pm \phi(x, y, t) \\
& \left.\left.+\phi_{2}(x, y)-\phi_{1}(x, y)\right]\right\}
\end{aligned}
$$

from which the time-varying phase $\phi(x, y, t)$ can be detected.

The Doppler shift frequency is

$$
\delta f=\frac{\phi(x, y, t)}{2 \pi t}=\frac{2 \mathrm{~d} z}{\lambda \mathrm{d} t}
$$

where $z$ is the displacement projected parallel to the line of sight

$z=\frac{\lambda}{2} \int_{t_{1}}^{t_{2}} \delta f \mathrm{~d} t$ 
This is the basic concept of the well-known incremental distance measuring interferometer. For a harmonically oscillating object $\phi(x, y, t)=(4 \pi \rho / \lambda) \cos (\Omega t)$, producing a frequency-modulated output signal at the detector with carrier frequency of $\Delta \omega / 2 \pi$ and amplitude and frequency of oscillation of $\rho$ and $\Omega$ respectively. The signal can be evaluated by common frequency analysis techniques.

In Fig. 14 a typical heterodyne arrangement for displacement and vibration analysis is shown. The arrangement can also be used for micro structure analysis with depth resolution in the subnanometer range and a lateral resolution of better than $1 \mu \mathrm{m}$ [9]. A microprofile is shown in Fig. 15.

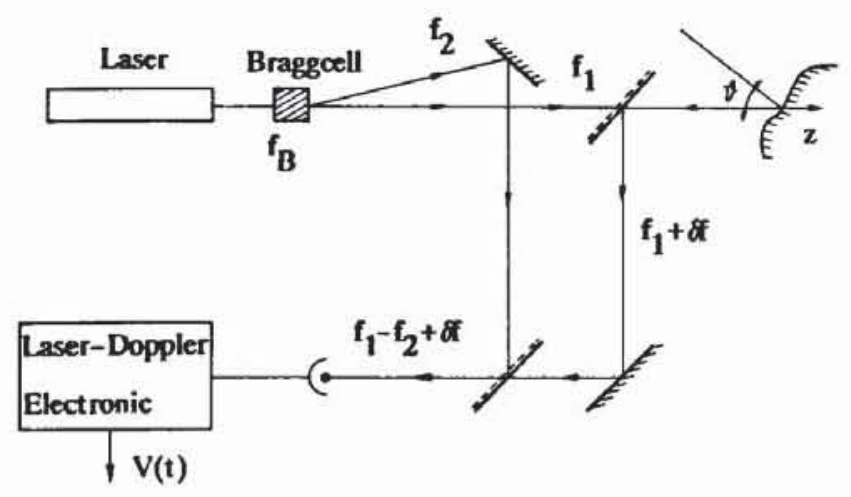

Fig. 14

Arrangement for two beam interferometry with a Bragg cell used for the constant frequency shift $f_{2}-f_{1}$

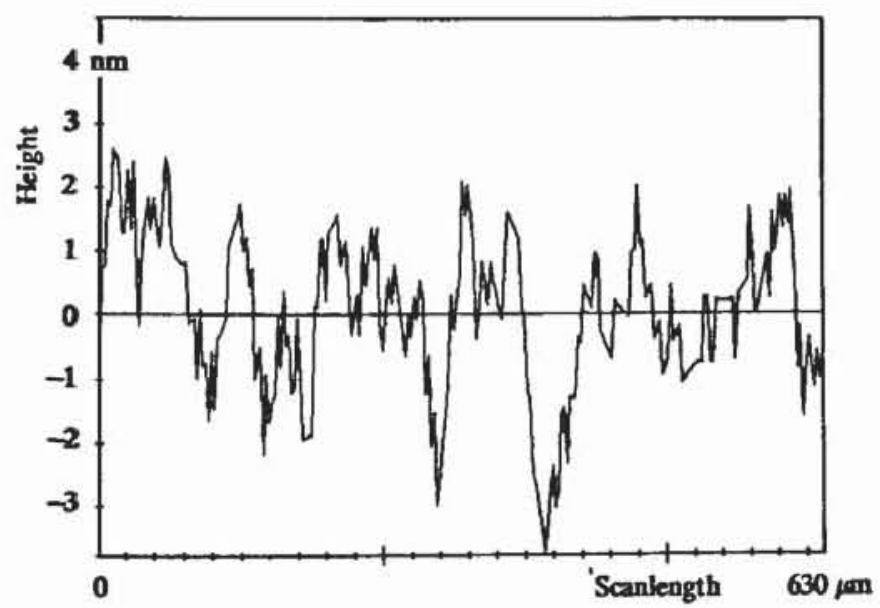

Fig, 15

Microprofile of a surface of polished zerodur, $R_{\mathrm{a}}=1 \mathrm{~nm}, R_{\mathrm{q}}=1.3 \mathrm{~nm}$

Heterodyne interferometry will lead to very useful future applications in precision measurements. For vibration analysis at given points, heterodyne interferometry gives not only the amplitude component of the vibration parallel to the line of sight, but also the frequency. In addition, it can be very useful for fringe analysis in holographic interferometry. It can be used to relate the holographic interference fringes to a known amplitude of vibration at a selected point. Furthermore, the heterodyne technique can be extended to speckle interferometry for vibration analysis.
By contrast, the laser Doppler velocimeter is used to measure flow of gases and liquids and the speed of optically rough surfaces with the best sensitivity perpendicular to the illumination and observation direction.

\section{Two Wavelength Interferometry}

Surface profiling is a useful application of interferometry where the object beam is focussed on an object that is scanned perpendicularly to the beam. The application of interferometry could be drastically increased, when the technique is extended to optically rough surfaces. In addition, with interferometry, phase measurement can only be determined with modulo $2 \pi$. An increase in the laser wavelength would be useful for the metrology of technical surfaces. Laser sources and the appropriate detectors are frequently not or not yet available. In addition, the high lateral resolution is lost by IR-laser wavelength.

In two-wavelength interferometry where the laser emits light with two slightly different wavelengths $\lambda_{1}$ and $\lambda_{2}$, the interferometer detects two separate interference patterns.

By an appropriate processing of the two individual interference patterns a new interference term of the form $\cos (4 \pi z / \lambda)$ is created where $\Lambda$ is an equivalent or beat wavelength given by

$\Lambda=\lambda_{1} \lambda_{2} /\left|\lambda_{1}-\lambda_{2}\right|$.

Since the wavelength difference $\left|\lambda_{1}-\lambda_{2}\right|$ is usually small, the equivalent wavelength is much larger than the original wavelength used. Since laser diodes can be easily tuned they are capable of generating a wide range of equivalent wavelengths, making them a good alternative to more expensive dye lasers or multi-frequency gas lasers.

The two-wavelengths used can either be time-multiplexed or can be present continuously. Furthermore, the twowavelength techniques can be applied in interferometry as well as in holography and speckleinterferometry.

\section{Two-Wavelength Heterodyne Interferometry}

Two-wavelength heterodyne interferometry can overcome some of the drawbacks of classical interferometry.

In a heterodyne interferometric set-up two waves are superposed to lead to an interference phenomenon. One of the two beams is frequency shifted by $f$.

The optical heterodyne signal is

$$
\begin{aligned}
I & =I_{0}[1+m \cos (2 \pi f t+\phi)] \\
& =I_{\mathrm{r}}+I_{\mathrm{s}}+2 \sqrt{I_{\mathrm{r}} I_{\mathrm{s}}} \cos (2 \pi f t+\phi) .
\end{aligned}
$$

$I_{\mathrm{r}}$ and $I_{\mathrm{s}}$ are the intensities of the reference and signal beams respectively.

The detected heterodyne signal is arranged to be shotnoise limited. There are different techniques to introduce the frequency shift such as using an acousto optical modulator (AOM) or a rotating grating or by using the 
Zeeman splitting in a laser cavity. In the DHI (double heterodyne interferometry methode) two heterodyne interference systems are superposed to lead to a beat frequency of the two wavelengths responsible for the distance measurement. The heterodyne signals $I_{\mathrm{h}}(t)$ are

$I_{\mathrm{h}}(t)=2 \sqrt{I_{r_{1}} I_{s_{1}}} \cos \left(2 \pi f_{1} t+\phi_{1}\right)+2 \sqrt{I_{r_{2}} I_{s_{2}}} \cos \left(2 \pi f_{2} t+\phi_{2}\right)$

$I_{r_{1}}, I_{s_{1}}, I_{r_{2}}, I_{s_{2}}$ are the intensities of the interfering reference and signal beams for the two wavelengths $\lambda_{1}$ and $\lambda_{2}, f_{1}$ and $f_{2}$ are the heterodyne frequencies

$\phi_{1}=\frac{4 \pi}{\lambda_{1}} z-2 \pi\left[\frac{v_{1}+f_{1}}{c}\right] L$

$\phi_{2}=\frac{4 \pi}{\lambda_{2}} z-2 \pi\left[\frac{v_{2}+f_{2}}{c}\right] L$

$z$ is the object distance to be measured and $c$ the velocity of the light, $v_{1}, v_{2}$ are the frequencies of the corresponding wavelengths $\lambda_{1}$ and $\lambda_{2}, L$ is the reference path. The heterodyne signal after the mixer is $I_{\text {sh }}(t)$ (superheterodyne signal)

$$
\begin{aligned}
I_{\mathrm{sh}}(t)= & 4 \sqrt{I_{r_{1}} I_{s_{1}} I_{r_{2}} I_{s_{2}}} \cos \left[2 \pi\left(f_{1}-f_{2}\right) t\right. \\
& \left.+\frac{4 \pi}{\Lambda} z-\frac{2 \pi}{c}\left(v_{1}+f_{1}-v_{2}-f_{2}\right) L\right]
\end{aligned}
$$

where

$\Lambda=\lambda_{1} \lambda_{2} /\left|\lambda_{1}-\lambda_{2}\right|$

In double heterodyne interferometry two laser wavelengths and two heterodyne frequencies are used simultaneously. A low frequency detection signal with a phase shift that corresponds to the effective wavelength is generated. A twowavelength double heterodyne interferometer (DHI) setup consists basically of two independent heterodyne interferometers working at different wavelengths $\lambda_{1}$ and $\lambda_{2}$ and different heterodyne frequencies $f_{1}$ and $f_{2}$. The phase of the beat frequency $f_{1}-f_{2}$ depends on the effective wavelength and can therefore be examined for distance evaluation as has been shown $[8,10]$.

The DHI is very appropriate for high precision absolute measurements. For the realization of a DHI there are, however, different possibilities. First two diode lasers giving $\lambda_{1}$ and $\lambda_{2}$ look very promising. An interesting way to obtain various wavelengths is to use a single laser diode in combination with a Bragg cell and two acousto optic modulators (AOM's).

In the case of a multiline laser to be discussed, the wavelengths $\lambda_{1}$ and $\lambda_{2}$ are perfectly combined and have a good relative stability. In a conventional setup (similar to the one shown in Fig. 14, but with a multiline laser instead of two lasers) the laser radiation would be first divided into target and reference beam.

\section{Double Heterodyne Interferometer with Rotational Matched Grating}

A two-wavelength heterodyne interferometry technique was developed for precision measurement. The heterodyne frequency difference for the two wavelengths was generated by a rotating grating for instance. After passing a lens $\mathrm{L}$, a polarizing beam splitter PBS and a quarterwave plate (QWP) the object beams are focussed on to the specimen under test by a microscopic objective (MO) (Fig. 16).

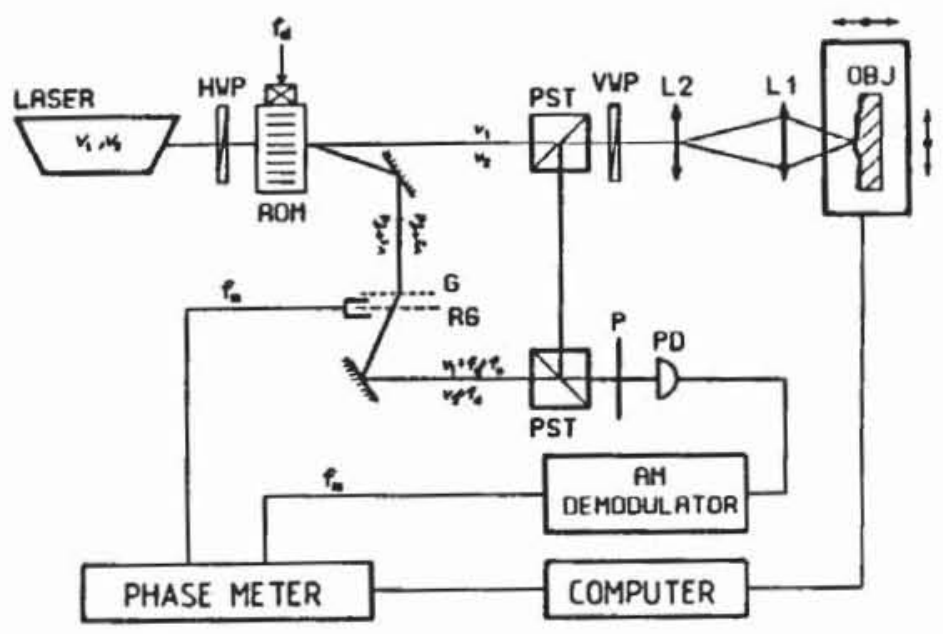

Fig. 16

Arrangement for $2 \lambda$-heterodyne interferometry with rotating grating

In the set-up shown in Fig. 16 the AOM is operated at driver frequency $f_{\mathrm{d}}$ leading to two frequencies in the first order diffracted beam for $\lambda_{1}$ of $v_{1}+f_{\mathrm{d}}$ and for $\lambda_{2}$ of $v_{2}+f_{\mathrm{d}}$. $\lambda_{1}$ and $\lambda_{2}$ are the wavelengths and $v_{1}, \nu_{2}$ the frequencies of the two wavelengths, $f_{\mathrm{d}}$ is the shifted frequency, which was introduced by the AOM.

The beams pass two diffraction gratings. The diffraction at the first grating (G) with a high spatial frequency $(600 \mathrm{lp} / \mathrm{mm})$ splits the two HeNe frequencies $v_{1}+f_{\mathrm{d}}$ and $v_{2}+f_{\mathrm{d}}$ where the first diffraction orders occur at $\alpha_{1}$ and $\alpha_{2}$. The grating was designed in such a way (and produced on photoresist) that the diffraction angle difference $\Delta \alpha$ between $v_{1}+f_{\mathrm{d}}$ and $v_{2}+f_{\mathrm{d}}$ is compensated at a second (low spatial frequency) grating. Therefore the first order diffraction of $v_{1}+f_{\mathrm{d}}$ and the zero order of $v_{2}+f_{\mathrm{d}}$, as well as the zero order of $v_{1}+f_{\mathrm{d}}$ and the minus first order of $v_{2}+f_{\mathrm{d}}$, become parallel as shown in Fig. 16.

The second grating (RG) is a standard low cost angle encoder (Hewlett Packard) with spatial frequency of $7.2 \mathrm{lp} / \mathrm{mm}$. It is continuously rotated by a motor. Due to a rotation of the grating, the first order diffracted light is frequency shifted by $f_{\mathrm{m}}$ while the zero order light passes the grating unaffected. Selecting one pair of parallel beams (as shown in Fig. 16) the frequencies of the beams are written 
as $v_{1}+f_{1}$ and $v_{2}+f_{2}$ where $f_{1}=f_{\mathrm{d}}+f_{\mathrm{m}}$ and $f_{2}=f_{\mathrm{d}}$. They serve as reference beams in the interferometer. The beat frequency $f_{\mathrm{m}}=f_{1}-f_{2}$ is generated due to the rotation of the angle encoder. In the experiment, the angle encoder wheel with $1000 \mathrm{lp} /$ revolution was rotated with 1200 revolutions/minute, the heterodyne beat frequency of $20 \mathrm{kHz}$ was found to be most appropriate. This frequency can be directly applied to a low frequency phase meter (e.g. two channel lock-in-amplifier LIA).

The reference for the lock-in-amplifier is directly taken from the angle encoder detector (square wave signal) output. Care must however be taken by the design of the encoder wheel when phase fluctuations in the reference path occur. The beat of the two heterodyne signals can be observed after demodulation and bandpass filtering, leading to

$U(t)=U_{0} \cos \left(2 \pi f_{\mathrm{m}} t-4 \pi z / \Lambda+\varphi\right)$.

Results were obtained with a $10 \times$ microscope objective and an avalanche diode as photodetector. The diffraction limited target spot was in the order of $3 \mu \mathrm{m}$. The clear working distance to the target was $6.5 \mathrm{~mm}$.

Two-wavelength double heterodyne interferometry has proven to be a powerful tool for accurate interferometric measurements on smooth as well as on optically rough surfaces. It is important especially for rough target surfaces to notice that the system behaves like a heterodyne interferometer with a synthetic wavelength $\Lambda=\lambda_{1} \lambda_{2} /\left|\lambda_{1}-\lambda_{2}\right|$. In the special arrangement the effective wavelength was $55.5 \mu \mathrm{m}$. The laser wavelengths themself were $632.8 \mathrm{~nm}$ and $640.1 \mathrm{~nm}$. It should be noticed that the roughness of the surface should be slightly smaller than the depth resolution expected. Fig. 17 shows the results of the measurement of a parabolic diamond turned metallic surface scanned in the $x$ direction.

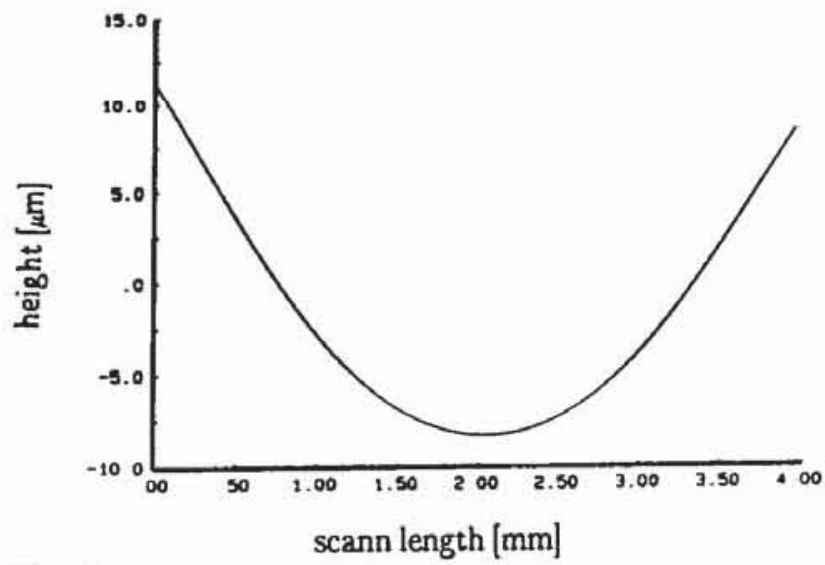

Fig. 17

Measurement of a parabolic mirror with $2 \lambda$-heterodyne interferometry, depth resolution $0.1 \mu \mathrm{m}$
Heterodyne interferometry is a powerful tool for high precision distance measurements and vibration analysis. Two-wavelength heterodyne techniques become very interesting for absolute distance measurement. It has been shown that a synthetic wavelength can be generated by two shorter ones, leading to techniques for measuring optically rougher surfaces. The theory is based on the assumption that the optical path difference is to be compared with the synthetic wavelength.

\section{Conclusion}

Different optical methods can be used for distance and topography measurement. The range and sensitivity need to be selected. The methods are contactless and fast. Interferometric methods are sometimes, however, too sensitive for environmental disturbances and for the measurement of technical objects. Furthermore surface roughness leads to unwanted Speckles to be taken care of by the analysis and information processing.

Therefore methods will be developed to measure topography of optically rough surfaces. Oblique incidence and $2 \lambda$ interferometry are possibilities for overcoming some of the limitations pointed out.

\section{References}

[1] K. J. Gasvik, Optical Metrology, Wiley, New York 1987.

[2] H.J. Tiziani, "Optical methods for precision measurements", Opt. Quantum Electr. 21, 253 (1989).

[3] H.J. Tiziani, in "Rechnerunterstützte Lasermeßtechnik", Technisches Messen 54, 221 (1987).

[4] P. Wieland and H.J. Tiziani, Proceedings Laser 93, Springer Verlag 1993.

[5] M. Takeda, H. Ina, and S. Kobayashi, J. Opt. Soc. Am. 72, 156 (1982).

[6] J.H. Brunning, in "Optical Shop Testing", ed. by D. Malacara, 409, Wiley, New York 1978.

[7] J. Schwider, R. Burow, K.E. Elsner, J. Grzonna, R. Spolaczyk, and K. Merkel, Appl. Opt. 22, 342 (1983).

[8] R. Dändliker, R. Thalmann, and D. Prongué, "Two-Wavelength Laser Interferometry Using Super-Heterodync Detcction", 14th Congress Int. Commission for Optics, Quebec, August 1987, paper B. 1.1. (SPIE Vol. 813).

[9] K. Leonhardt, K.-H. Rippert, and H. J. Tiziani, in "Optische Profilometrie und Rauheitsmessung". Technisches Messen 54, 243 (1987).

[10] Z. Sodnik, E. Fischer, Th. Ittner, and H.J. Tiziani, "Twowavelength double heterodyne Interferometry using a matched grating technique", Appl. Opt. 30, 3139 (1991).

[11] B. Dörband and H. J. Tiziani, Appl. Opt. 24, 2604 (1985).

Presented at the Discussion Meeting of the Deutsche Bunsen-Gesellschaft für Physikalische Chemie "Laser Diagnostics for Industrial Processes" in Heidelberg, June 28th to July 2nd, 1993 